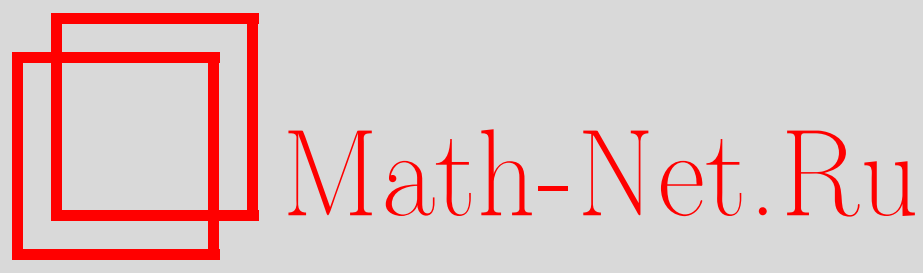

А. М. Левин, М. А. Ольшанецкий, А. В. Зотов, Квантовые $R$-матрицы Бакстера-Белавина и многомерные пары Лакса для уравнения Пенлеве VI, ТМФ, 2015, том 184, номер 1, 41-56

DOI: https://doi.org/10.4213/tmf8877

Использование Общероссийского математического портала Math-Net.Ru подразумевает, что вы прочитали и согласны с пользовательским соглашением http: //www.mathnet.ru/rus/agreement

Параметры загрузки:

IP : 54.89 .56 .158

26 апреля 2023 г., 13:36:51

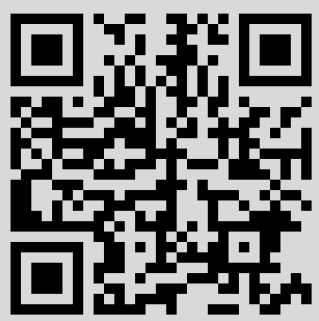




\title{
КВАНТОВЫЕ $R$-МАТРИЦЫ БАКСТЕРА-БЕЛАВИНА И МНОГОМЕРНЫЕ ПАРЫ ЛАКСА ДЛЯ УРАВНЕНИЯ ПЕНЛЕВЕ VI
}

\begin{abstract}
Квантовые эллиптические $R$-матрицы удовлетворяют ассоциативному уравнению Янга-Бакстера в $\operatorname{Mat}(N)^{\otimes 2}$, которое можно рассматривать как некоммутативный аналог тождеств Фэя для скалярной функции Кронекера. Представлен более широкий список $R$-матричнозначных тождеств для эллиптических функций. В частности, предложен аналог тождеств Фэя в пространстве $\operatorname{Mat}(N)^{\otimes 2}$, а также описано представление для $R$-матриц в виде двойных рядов Кронекера. В качестве приложения с помощью $\left(\mathbb{Z}_{N} \times \mathbb{Z}_{N}\right)$-симметричной эллиптической $R$-матрицы построены $R$-матричнозначные $\left(2 N^{2} \times 2 N^{2}\right)$-пары Лакса для уравнения Пенлеве VI (в эллиптической форме) с четырьмя произвольными константами. Более точно, случай четырех произвольных констант отвечает нечетным $N$, а четные $N$ соответствуют случаю единственной константы в уравнении.
\end{abstract}

Ключевые слова: квантовые $R$-матрицы, многомерные пары Лакса, уравнения Пенлеве.

DOI: $10.4213 / \operatorname{tmf} 8877$

\section{1. ВВЕДЕНИЕ И ОСНОВНЫЕ РЕЗУЛЬТАТЫ}

В настоящей работе мы продолжаем изучение тождеств для квантовых (и классических) $R$-матриц, аналогичных тождествам для скалярных эллиптических функций [1], [2]. Предложен аналог тождества Фэя в пространстве $\operatorname{Mat}(N)^{\otimes 2}$. Он позволяет для уравнения Пенлеве VI построить многомерные пары Лакса с матричными элементами в виде квантовых $R$-матриц.

* Факультет математики, Национальный исследовательский университет - Высшая школа экономики, Москва, Россия. E-mail: alevin@hse.ru

${ }^{\dagger}$ Институт теоретической и экспериментальной физики, Москва, Россия. E-mail: olshanet@itep.ru

${ }^{\ddagger}$ Московский физико-технический институт, Долгопрудный, Московская обл., Россия

$\S$ Математический институт им. В. А. Стеклова РАН, Москва, Россия.

E-mail: zotov@mi.ras.ru 
Начнем со списка свойств и тождеств для эллиптических функций, а затем приведем их $R$-матричные аналоги. Большая часть свойств известна из работ [1]-[7].

Рассмотрим следующие функции:

$$
\begin{gathered}
\phi(z, u)=\frac{\vartheta^{\prime}(0) \vartheta(z+u)}{\vartheta(z) \vartheta(u)}, \\
E_{1}(z)=\frac{\vartheta^{\prime}(z)}{\vartheta(z)}, \quad E_{2}(z)=-\partial_{z} E_{1}(z)=\wp(z)-\frac{1}{3} \frac{\vartheta^{\prime \prime \prime}(0)}{\vartheta^{\prime}(0)},
\end{gathered}
$$

где $\vartheta(z)$ - нечетная тета-функция,

$$
\vartheta(z)=\vartheta(z \mid \tau)=\sum_{k \in \mathbb{Z}} \exp \left\{\pi i \tau\left(k+\frac{1}{2}\right)^{2}+2 \pi i\left(z+\frac{1}{2}\right)\left(k+\frac{1}{2}\right)\right\},
$$

а $\wp(z)-\wp$-функция Вейерштрасса.

Следуя монографии [8], функцию (1.1) будем называть функцией Кронекера, а функции (1.2) называются (первой и второй) функциями Эйзенштейна. Функцию Кронекера можно рассматривать как сечение расслоения Пуанкаре $\mathcal{P}$ над $\Sigma_{\tau} \times \Sigma_{\tau}^{\prime}$. Здесь $\Sigma_{\tau}-$ эллиптическая кривая,

$$
\Sigma_{\tau}=\mathbb{C} /(\mathbb{Z}+\tau \mathbb{Z}), \quad \operatorname{Im} \tau>0,
$$

а $\Sigma_{\tau}^{\prime}$ - ее якобиан $\left(\Sigma_{\tau}^{\prime} \sim \Sigma_{\tau}\right)$. Расслоение Пуанкаре $\mathcal{P}$ есть линейное расслоение над $\Sigma_{\tau} \times \Sigma_{\tau}^{\prime}$

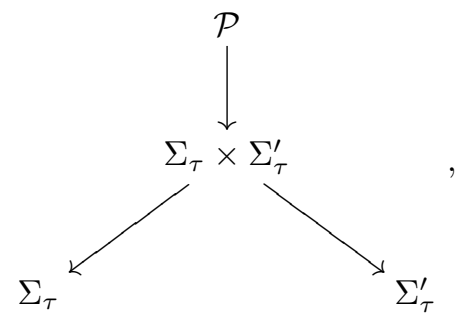

заданное соотношениями (1.6), (1.7), (1.10) и (1.11), приведенными ниже.

Свойства тета-функции (1.3) (включая тождества Римана [9]) приводят к следующему набору свойств и взаимосвязей для функций (1.1), (1.2).

- Симметрия аргументов:

$$
\phi(z, u)=\phi(u, z), \quad z \in \Sigma_{\tau}, \quad u \in \Sigma_{\tau}^{\prime} .
$$

- Локальное разложение:

$$
\phi(z, u)=\frac{1}{z}+E_{1}(u)+\frac{z}{2}\left(E_{1}^{2}(u)-\wp(u)\right)+O\left(z^{2}\right) .
$$

- Bъичет:

$$
\operatorname{Res}_{z=0} \phi(z, u)=\operatorname{Res}_{u=0} \phi(z, u)=\operatorname{Res}_{z=0} E_{1}(z)=1 .
$$

- Четность:

$$
\phi(-z,-u)=-\phi(z, u), \quad E_{1}(-z)=-E_{1}(z), \quad E_{2}(-z)=E_{2}(z) .
$$


- (Квази)периодические свойства:

$$
\begin{array}{lll}
\phi(z+1, u)=\phi(z, u), & E_{1}(z+1)=E_{1}(z), & E_{2}(z+1)=E_{2}(z), \\
\phi(z+\tau, u)=e^{-2 \pi i u} \phi(z, u), & E_{1}(z+\tau)=E_{1}(z)-2 \pi i, & E_{2}(z+\tau)=E_{2}(z) .
\end{array}
$$

- Уравнение теплопроводности:

$$
2 \pi i \partial_{\tau} \phi(z, u)=\partial_{z} \partial_{u} \phi(z, u)
$$

- Производные:

$$
\begin{aligned}
& \partial_{u} \phi(z, u)=\phi(z, u)\left(E_{1}(z+u)-E_{1}(u)\right), \\
& \partial_{z} \phi(z, u)=\phi(z, u)\left(E_{1}(z+u)-E_{1}(z)\right) .
\end{aligned}
$$

- Тождество Фэя (тройной секущей) [10]:

$$
\phi(x, u) \phi(y, w)=\phi(x-y, u) \phi(y, u+w)+\phi(y-x, w) \phi(x, u+w) .
$$

- Вырожденные тождества Фэя:

$$
\phi(x, z) \phi(x, w)=\phi(x, z+w)\left(E_{1}(x)+E_{1}(z)+E_{1}(w)-E_{1}(x+z+w)\right)
$$

или

$$
\begin{aligned}
\phi(x, z) \phi(y, z) & =\phi(x+y, z)\left(E_{1}(x)+E_{1}(y)+E_{1}(z)-E_{1}(x+y+z)\right), \\
\phi(x, z) \phi(x,-z) & =E_{2}(x)-E_{2}(z)=\wp(x)-\wp(z) .
\end{aligned}
$$

- Геометрическая интерпретация. Функция Кронекера $\phi(z, u)$ является сечением расслоения Пуанкаре $\mathcal{P}$. Это линейное расслоение над $\Sigma_{\tau} \times \Sigma_{\tau}$, заданное условиями (1.6), (1.7), (1.10), (1.11).

- Функиия Грина. Функция Кронекера является функцией Грина оператора $\bar{\partial}$ в пространстве 1 -форм $\mathcal{A}^{(1,0)}\left(\Sigma_{\tau}\right)$ с граничными условиями $(1.10)$ и $(1.11)$ :

$$
\bar{\partial} \phi(z, u)=\delta^{2}(z, \bar{z}) .
$$

(. Квантовые $\$ R \$$-матрицы)Квантовые $R$-матрицы Рассмотрим $\left(\mathbb{Z}_{N} \times \mathbb{Z}_{N}\right)$-симметричную эллиптическую $R$-матрицу (Бакстера-Белавина [3], [4]) в фундаментальном представлении (см. также работы [5]). Она определяется в терминах конечномерного представления группы Гейзенберга. Пусть $Q, \Lambda \in \operatorname{Mat}(N, \mathbb{C})$,

$$
\begin{gathered}
Q_{k l}=\delta_{k l} e^{2 \pi i k / N}, \quad \Lambda_{k l}=\delta_{k-l+1,0(\bmod N)}, \quad k, l=1, \ldots, N, \\
e^{2 \pi i \gamma_{1} \gamma_{2} / N} Q^{\gamma_{1}} \Lambda^{\gamma_{2}}=\Lambda^{\gamma_{2}} Q^{\gamma_{1}}, \quad \gamma_{1}, \gamma_{2} \in \mathbb{Z} .
\end{gathered}
$$

Определим базис синус-алгебры в $\operatorname{Mat}(N, \mathbb{C})$ :

$$
T_{\gamma}:=T_{\gamma_{1} \gamma_{2}}=e^{\pi i \gamma_{1} \gamma_{2} / N} Q^{\gamma_{1}} \Lambda^{\gamma_{2}}, \quad \gamma_{1}, \gamma_{2}=0,1, \ldots, N-1 .
$$

Такое же определение сохраним и для всех $\gamma \in \mathbb{Z} \times \mathbb{Z}$. Тогда имеем

$$
T_{\alpha} T_{\beta}=\kappa_{\alpha, \beta} T_{\alpha+\beta}, \quad \kappa_{a, b}=e^{\pi i\left(\beta_{1} \alpha_{2}-\beta_{2} \alpha_{1}\right) / N},
$$

где $\alpha+\beta=\left(\alpha_{1}+\beta_{1}, \alpha_{2}+\beta_{2}\right)$. 
$R$-матрица задается формулой

$$
R_{12}^{\hbar}(u)=\sum_{\alpha \in \mathbb{Z}_{N} \times \mathbb{Z}_{N}} \varphi_{\alpha}\left(u, \omega_{\alpha}+\hbar\right) T_{\alpha} \otimes T_{-\alpha} \in \operatorname{Mat}(N, \mathbb{C})^{\otimes 2},
$$

где $\mathrm{e}^{1)}$

$$
\varphi_{\alpha}\left(u, \omega_{\alpha}+\hbar\right)=e^{2 \pi i u \partial_{\tau} \omega_{\alpha}} \phi\left(u, \omega_{\alpha}+\hbar\right), \quad \omega_{\alpha}=\frac{\alpha_{1}+\alpha_{2} \tau}{N} .
$$

Свойство $\left(\mathbb{Z}_{N} \times \mathbb{Z}_{N}\right)$-симметрии означает, что для $g=Q, \Lambda$ выполняется равенство

$$
g \otimes g R_{12}^{\hbar}(u) g^{-1} \otimes g^{-1}=R_{12}^{\hbar}(u) .
$$

В случае $N=1 R$-матрица (1.24) есть скалярная функция Кронекера $\phi(\hbar, u)(1.1)$. Отметим, что матрица (1.24) нормирована таким образом, что условие унитарности имеет вид

$$
R_{12}^{\hbar}(u) R_{21}^{\hbar}(-u)=N^{2} \phi(N \hbar, u) \phi(N \hbar,-u) 1 \otimes 1=N^{2}(\wp(N \hbar)-\wp(u)) 1 \otimes 1 .
$$

Его можно рассматривать как аналог равенства (1.18). Здесь $R_{21}(z)=P_{12} R_{12}(z) P_{12}$, где

$$
P_{12}=\frac{1}{N} \sum_{\alpha} T_{\alpha} \otimes T_{-\alpha}=\sum_{i, j=1}^{N} E_{i j} \otimes E_{j i}, \quad\left(E_{i j}\right)_{k l}=\delta_{i k} \delta_{j l},
$$

есть оператор перестановки.

Мы также используем обозначение $R_{a b}^{\hbar}(z)$, отличающееся от (1.27) тем, что вместо $T_{\alpha} \otimes T_{-\alpha}$ подставлено $T_{\alpha}^{a} \otimes T_{-\alpha}^{b}=1 \otimes \cdots 1 \otimes T_{\alpha} \otimes 1 \cdots 1 \otimes T_{-\alpha} \otimes 1 \cdots \otimes 1$ (здесь $T_{\alpha}$ и $T_{-\alpha}$ - матрицы в $a$-й и $b$-й компонентах тензорного произведения). Количество компонент в тензорном произведении есть целое число $\widetilde{N}$. Это означает, что $R_{a b}^{\hbar}$ является элементом пространства $\operatorname{Mat}(N, \mathbb{C})^{\otimes \widetilde{N}}$, т. е. $\left(N^{\widetilde{N}} \times N^{\widetilde{N}}\right)$-матрицей.

Свойства и тождества (1.6)-(1.19) имеют следующие $R$-матричные аналоги.

- Симметрия аргументов:

$$
R_{12}^{\hbar}(z)=R_{12}^{z / N}(N \hbar) P_{12} .
$$

- Локальное разложение по $ћ$ представляет собой классический предел:

$$
R_{12}^{\hbar}(z)=\hbar^{-1} 1 \otimes 1+r_{12}(z)+\hbar m_{12}(z)+O\left(\hbar^{2}\right),
$$

где $r_{12}(z)$ - классическая $r$-матрица (Белавина-Дринфельда [4]),

$$
r_{12}(z)=E_{1}(z) 1 \otimes 1+\sum_{\alpha \neq 0} \varphi_{\alpha}(z) T_{\alpha} \otimes T_{-\alpha}
$$

a

$$
m_{12}(z)=\frac{E_{1}^{2}(z)-\wp(z)}{2} 1 \otimes 1+\left.\sum_{\alpha \neq 0} e^{2 \pi i z \partial_{\tau} \omega_{\alpha}} \partial_{u} \phi(z, u)\right|_{u=\omega_{\alpha}} T_{\alpha} \otimes T_{-\alpha} .
$$

Аналогично соотношению (1.7) имеем

$$
r_{12}^{2}(z)-2 m_{12}(z)=1 \otimes 1 N^{2} \wp(z),
$$

1) Здесь $\partial_{\tau} \omega_{\alpha}=\alpha_{2} / N$. 
т. е. квантовая $R$-матрица есть матричный аналог функции Кронекера (1.1), а классическая - матричный аналог первой функции Эйзенштейна (1.2). Разложение по z (в окрестности $z=0$ ) имеет вид

$$
R_{12}^{\hbar}(z)=\frac{N P_{12}}{z}+R_{12}^{\hbar,(0)}+O(z)
$$

где $\mathrm{e}^{2)}$

$$
R_{12}^{\hbar,(0)}=\sum_{\alpha} T_{\alpha} \otimes T_{-\alpha}\left(E_{1}\left(\hbar+\omega_{\alpha}\right)+2 \pi i \partial_{\tau} \omega_{\alpha}\right)
$$

- Bъчеть:

$$
\operatorname{Res}_{\hbar=0} R_{12}^{\hbar}(z)=1 \otimes 1, \quad \operatorname{Res}_{z=0} R_{12}^{\hbar}(z)=\operatorname{Res}_{z=0} r_{12}(z)=N P_{12} .
$$

- Четность:

$$
R_{12}^{\hbar}(z)=-R_{21}^{-\hbar}(-z), \quad r_{12}(z)=-r_{21}(-z), \quad m_{12}(z)=m_{21}(-z) .
$$

$R$-матричный аналог функции $E_{2}(u)=E_{2}(-u)(1.2)$ появляется в виде функции $F_{12}^{0}(u)=-\partial_{u} r_{12}(u)$ (что естественно, поскольку $r_{12}(u)$ есть матричный аналог функции $\left.E_{1}(u)\right)$. Классическая $r$-матрица нечетна. Следовательно, $F_{12}^{0}(u)$ четная. Такой же ответ следует и из локального разложения (1.7), (1.30): $E_{2}(u)=-\left.\partial_{u} \phi(z, u)\right|_{z=0}$, тогда $-\left.\partial_{u} R_{12}^{z}(u)\right|_{z=0}=-\partial_{u} r_{12}(u)$.

- (Квази)периодические свойства:

$$
\begin{aligned}
R_{12}^{\hbar}\left(z+N \omega_{\gamma}\right) & =e^{-2 \pi i N \hbar \partial_{\tau} \omega_{\gamma}} T_{\gamma}^{-1} \otimes 1 R_{12}^{\hbar}(z) T_{\gamma} \otimes 1 \\
R_{12}^{\hbar+\omega_{\gamma}}(z) & =e^{-2 \pi i z \partial_{\tau} \omega_{\gamma}} T_{\gamma}^{-1} \otimes 1 R_{12}^{\hbar}(z)\left(1 \otimes T_{\gamma}\right) .
\end{aligned}
$$

В частности,

$$
\begin{gathered}
R_{12}^{\hbar}(z+1)=Q^{-1} \otimes 1 R_{12}^{\hbar}(z) Q \otimes 1, \\
R_{12}^{\hbar}(z+\tau)=e^{-2 \pi i \hbar} \Lambda^{-1} \otimes 1 R_{12}^{\hbar}(z) \Lambda \otimes 1 \\
R_{12}^{\hbar+1}(z)=R_{12}^{\hbar}(z), \quad R_{12}^{\hbar+\tau}(z)=e^{-2 \pi i z} R_{12}^{\hbar}(z), \\
r_{12}(z+1)=Q^{-1} \otimes 1 r_{12}(z) Q \otimes 1, \\
r_{12}(z+\tau)=\Lambda^{-1} \otimes 1 r_{12}(z) \Lambda \otimes 1-2 \pi i 1 \otimes 1 .
\end{gathered}
$$

Перепишем равенство (1.39) в виде

$$
\begin{aligned}
& R_{a b}^{\hbar+1 / N}\left(z_{a}-z_{b}\right)=Q_{a}^{-1} R_{a b}^{\hbar}\left(z_{a}-z_{b}\right) Q_{b} \\
& R_{a b}^{\hbar+\tau / N}\left(z_{a}-z_{b}\right)=e^{-2 \pi i\left(z_{a}-z_{b}\right) / N} \Lambda_{a}^{-1} R_{a b}^{\hbar}\left(z_{a}-z_{b}\right) \Lambda_{b} .
\end{aligned}
$$

$R$-матричнозначная матрица Лакса для $g l_{\widetilde{N}}$-системы Калоджеро-Мозера [1] имеет вид

$$
\mathcal{L}(\hbar)=\sum_{a, b=1}^{\widetilde{N}} \widetilde{\mathrm{E}}_{a b} \otimes \mathcal{L}_{a b}(\hbar), \quad \mathcal{L}_{a b}(\hbar)=\delta_{a b} p_{a} 1_{a} \otimes 1_{b}+\nu\left(1-\delta_{a b}\right) R_{a b}^{\hbar}\left(z_{a}-z_{b}\right),
$$

2) Матрица $R_{12}^{\hbar,(0)}$ появляется в описании релятивистского волчка как часть обратного тензора инерции [11]. 
где $\widetilde{\mathrm{E}}_{a b}$ - стандартный базис в $g l_{\widetilde{N}}:\left(\widetilde{\mathrm{E}}_{a b}\right)_{c d}=\delta_{a c} \delta_{b d}, a, b, c, d=1, \ldots, \tilde{N}$. Тогда из формул (1.43), (1.44) следует, что

$$
\begin{aligned}
& \mathcal{L}\left(\hbar+\frac{1}{N}\right)=\mathbf{Q}^{-1} \mathcal{L}(\hbar) \mathbf{Q}, \\
& \mathcal{L}\left(\hbar+\frac{\tau}{N}\right)=e^{-\mathbf{Z} / N} \boldsymbol{\Lambda}^{-1} \mathcal{L}(\hbar) \boldsymbol{\Lambda} e^{\mathbf{Z} / N},
\end{aligned}
$$

где

$$
\mathbf{Q}=\bigoplus_{a=1}^{\tilde{N}} Q_{a}, \quad \mathbf{\Lambda}=\bigoplus_{a=1}^{\tilde{N}} \Lambda_{a}, \quad \mathbf{Z}=\bigoplus_{a=1}^{\tilde{N}} z_{a} 1_{a}
$$

суть блочно-диагональные матрицы. Количество блоков равно $\widetilde{N} \times \widetilde{N}$, а размер каждого блока равен $N^{\widetilde{N}} \times N^{\widetilde{N}}$.

- Уравнение теплопроводности:

$$
2 \pi i \partial_{\tau} R_{12}^{\hbar}(z)=\partial_{z} \partial_{\hbar} R_{12}^{\hbar}(z)
$$

- Производние

$$
\begin{aligned}
\partial_{\hbar} R_{12}^{\hbar}(z)= & \frac{1}{2}\left(r_{12}(z+N \hbar) R_{12}^{\hbar}(z)+R_{12}^{\hbar}(z) r_{12}(z-N \hbar)\right)+ \\
& +\frac{N}{2}\left(E_{1}(z+N \hbar)-E_{1}(z-N \hbar)-2 E_{1}(N \hbar)\right) R_{12}^{\hbar}(z), \\
\partial_{z} R_{12}^{\hbar}(z)= & \frac{1}{2 N}\left(r_{12}(z+N \hbar) R_{12}^{\hbar}(z)-R_{12}^{\hbar}(z) r_{12}(z-N \hbar)\right)+ \\
& +\frac{1}{2}\left(E_{1}(z+N \hbar)+E_{1}(z-N \hbar)-2 E_{1}(z)\right) R_{12}^{\hbar}(z) .
\end{aligned}
$$

- Тождество Фэя в пространстве $\operatorname{Mat}(N, \mathbb{C})^{\otimes 3}[1],[12]$, [2]:

$$
R_{a b}^{\hbar} R_{b c}^{\hbar^{\prime}}=R_{a c}^{\hbar^{\prime}} R_{a b}^{\hbar-\hbar^{\prime}}+R_{b c}^{\hbar^{\prime}-\hbar} R_{a c}^{\hbar}, \quad R_{a b}^{\hbar}=R_{a b}^{\hbar}\left(z_{a}-z_{b}\right)
$$

Обе части тождества - элементы пространства $\operatorname{Mat}(N, \mathbb{C})^{\otimes 3}$. Оно использовалось в работе [1] для построения многомерных пар Лакса для системы Калоджеро. Здесь мы докажем аналог того же тождества $(1.15)$ в пространстве $\operatorname{Mat}(N, \mathbb{C})^{\otimes 2}$.

- Тождество Фэя в пространстве $\operatorname{Mat}(N, \mathbb{C})^{\otimes 2}$ :

$$
\begin{aligned}
R_{12}^{\hbar}(z) R_{21}^{\hbar^{\prime}}(-w)= & N \phi\left(N \hbar^{\prime}, \frac{z-w}{N}+\hbar^{\prime}-\hbar\right) R_{12}^{\hbar-\hbar^{\prime}}\left(z+N \hbar^{\prime}\right)- \\
& -N \phi\left(N \hbar, \frac{z-w}{N}+\hbar^{\prime}-\hbar\right) R_{12}^{\hbar-\hbar^{\prime}}(w+N \hbar)+ \\
& +N \phi\left(-w, \frac{z-w}{N}+\hbar^{\prime}-\hbar\right) R_{12}^{(z-w) / N}(w+N \hbar)- \\
& -N \phi\left(-z, \frac{z-w}{N}+\hbar^{\prime}-\hbar\right) R_{12}^{(z-w) / N}\left(z+N \hbar^{\prime}\right) .
\end{aligned}
$$

3) Тождества для производных от $R$-матрицы по постоянной Планка и спектральному параметру были найдены в работах [6] и [7] соответственно. Авторы этих работ использовали другую нормировку $R$-матрицы. 
Скалярный аналог этого тождества получается следующим образом: применим соотношение $(1.15)$ (с $\left.x=\hbar, y=\hbar^{\prime}\right)$ к $\phi(\hbar, z) \phi\left(\hbar^{\prime},-w\right)$, а затем применим (1.15) еще раз в полученном выражении. В итоге получим скалярный аналог правой части (1.52).

- Вырожденные тождества Фэя (1.51) в пространстве $\operatorname{Mat}(N, \mathbb{C})^{\otimes 3}$ :

$$
\begin{aligned}
R_{a b}^{\hbar} R_{b c}^{\hbar} & =R_{a c}^{\hbar} r_{a b}+r_{b c} R_{a c}^{\hbar}-\partial_{\hbar} R_{a c}^{\hbar} \\
R_{a b}^{\hbar}(z) R_{b c}^{\hbar^{\prime}}(-z) & =R_{a c}^{\hbar^{\prime},(0)} R_{a b}^{\hbar-\hbar^{\prime}}(z)+R_{b c}^{\hbar^{\prime}-\hbar}(-z) R_{a c}^{\hbar,(0)}+N F_{b c}^{\hbar^{\prime}-\hbar}(-z) P_{a c}
\end{aligned}
$$

где $F_{a b}^{\hbar}(u)=\partial_{u} R_{a b}^{\hbar}(u)$ и $R_{a b}^{\hbar,(0)}$ задана в $(1.34),(1.35)$.

- Вырожденные тождества Фэя (1.52) в пространстве $\operatorname{Mat}(N, \mathbb{C})^{\otimes 2}$ :

$$
\begin{aligned}
& R_{12}^{\hbar}(z) R_{21}^{\hbar}(-w)=N \phi\left(\frac{z-w}{N}, N \hbar\right)\left(r_{12}(z+N \hbar)-r_{12}(w+N \hbar)\right)+ \\
& \quad+N \phi\left(\frac{w-z}{N}, z\right) R_{12}^{(z-w) / N}(z+N \hbar)-N \phi\left(\frac{w-z}{N}, w\right) R_{12}^{(z-w) / N}(w+N \hbar)+ \\
& \quad+N^{2} 1 \otimes 1 \phi\left(\frac{z-w}{N}, N \hbar\right)\left(E_{1}(N \hbar)-E_{1}\left(N \hbar+\frac{z-w}{N}\right)\right), \\
& R_{12}^{\hbar}(z) R_{21}^{\hbar^{\prime}}(-z)=N \phi\left(\hbar^{\prime}-\hbar,-z\right)\left(r_{12}(z+N \hbar)-r_{12}\left(z+N \hbar^{\prime}\right)\right)- \\
& \quad-N \phi\left(\hbar^{\prime}-\hbar, N \hbar\right) R_{12}^{\hbar-\hbar^{\prime}}(z+N \hbar)+N \phi\left(\hbar^{\prime}-\hbar, N \hbar^{\prime}\right) R_{12}^{\hbar-\hbar^{\prime}}\left(z+N \hbar^{\prime}\right)+ \\
& \quad+N^{2} 1 \otimes 1 \phi\left(\hbar^{\prime}-\hbar,-z\right)\left(E_{1}(z)-E_{1}\left(z+\hbar-\hbar^{\prime}\right)\right) .
\end{aligned}
$$

- Геометрическая интерпретация: в силу квазипериодических свойств, заданных в (1.38)-(1.41), $R$-матрица имеет следующую геометрическую интерпретацию. Пусть $V_{1}\left(V_{2}\right)$ - векторное расслоение ранга $N$ и степени один над эллиптической кривой $\Sigma_{\tau}^{(1)}$ с координатой $z_{1}$ (кривой $\Sigma_{\tau}^{(2)}$ с координатой $z_{2}$ ). Рассмотрим расслоение $V_{1} \otimes V_{2}$ над $\Sigma_{\tau}^{(1)} \times \Sigma_{\tau}^{(2)}$. Пусть $\operatorname{Aut}_{\mathrm{PGL}(N)}\left(V_{1} \otimes V_{2}\right)-$ группа автоморфизмов расслоения (калибровочная группа). Сечения $\Gamma\left(\operatorname{Aut}_{\mathrm{PGL}(N)}\left(V_{1} \otimes V_{2}\right)\right)$ зависят только от антидиагональной части $\widetilde{\Sigma}_{\tau}$ произведения $\Sigma_{\tau}^{(1)} \times \Sigma_{\tau}^{(2)}$ с координатой $z=z_{1}-z_{2}$. Пусть $\widetilde{\Sigma}_{\tau}^{\prime}$ - дуальная кривая с координатой $\hbar$, а $\mathcal{P}$ - расслоение Пуанкаре $\mathcal{P}$ над $\widetilde{\Sigma}_{\tau} \times \widetilde{\Sigma}_{\tau}^{\prime}(1.5)$. Тогда $R$-матрица (1.24) является сечением расслоения

$$
R_{12}^{\hbar}(z) \in \Gamma\left(\left(\operatorname{Aut}_{\mathrm{PGL}(N)}\left(V_{1} \otimes V_{2}\right)\right) \otimes \mathcal{P}\right)
$$

- Функция Грина: аналогично (1.19) $R$-матрицу можно рассматривать как функцию Грина оператора $\bar{\partial}$ :

$$
\bar{\partial} R_{12}^{\hbar}(z)=N P_{12} \delta^{2}(z, \bar{z})
$$

Свойства (1.30)-(1.48) несложно выводятся из их скалярных аналогов, за исключением равенства (1.33), которое следует из условия унитарности (1.27) в классическом пределе (1.30). Тождества для производных (1.49), (1.50) были получены в работах [6], [7]. Вырожденные тождества $(1.53),(1.54)$ в пространстве $\operatorname{Mat}(N, \mathbb{C})^{\otimes 3}$ следуют из невырожденного тождества (1.51) и локальных разложений $(1.30),(1.34)$.

Наибольший интерес (в настоящей работе) представляет тождество Фэя (1.52) в пространстве $\operatorname{Mat}(N, \mathbb{C})^{\otimes 2}$ и его вырождения $(1.55),(1.56)$. Мы докажем эти тождества ниже. Доказательство основано на свойстве (1.29) "симметрии аргументов" и использовании скалярных тождеств Фэя (1.15)-(1.17). 
Уравнение Пенлеве VI. В качестве приложения полученных формул мы предлагаем конструкцию многомерных пар Лакса для уравнения Пенлеве VI. Обозначим полупериоды эллиптической кривой $\Sigma_{\tau}$ через $\Omega_{a}, a=0,1,2,3$ :

$$
\left\{\Omega_{0}, \Omega_{1}, \Omega_{2}, \Omega_{3}\right\}=\{0,1 / 2,(1+\tau) / 2, \tau / 2\} .
$$

Уравнение Пенлеве VI в эллиптической форме [13] имеет вид

$$
\frac{d^{2} u}{d \tau^{2}}=-\sum_{a=0}^{3} \nu_{a}^{2} \wp^{\prime}\left(u+\Omega_{a}\right)
$$

Пусть $N$ - нечетное натуральное число. Рассмотрим следующую пару блочных матриц ${ }^{4)}$ :

$$
\begin{aligned}
L(\hbar) & =\frac{1}{2} \frac{d u}{d \tau}\left(\begin{array}{cc}
1 \otimes 1 & 0 \\
0 & -1 \otimes 1
\end{array}\right)+\sum_{a=0}^{3} \frac{\nu_{a}}{N \sqrt{-2}}\left(\begin{array}{cc}
0 & \mathcal{R}_{12}^{\hbar, a}(u) \\
\mathcal{R}_{21}^{\hbar, a}(-u) & 0
\end{array}\right), \\
M(\hbar) & =\sum_{a=0}^{3} \frac{\nu_{a}}{N \sqrt{-2}}\left(\begin{array}{cc}
0 & \mathcal{F}_{12}^{\hbar, a}(u) \\
\mathcal{F}_{21}^{\hbar, a}(-u) & 0
\end{array}\right),
\end{aligned}
$$

где

$$
\begin{gathered}
\mathcal{R}_{12}^{\hbar, a}(u)=e^{2 \pi i N \hbar \partial_{\tau} \Omega_{a}} R_{12}^{\hbar}\left(u+N \Omega_{a}\right), \\
\mathcal{R}_{21}^{\hbar, a}(-u)=e^{-2 \pi i N \hbar \partial_{\tau} \Omega_{a}} R_{21}^{\hbar}\left(-u-N \Omega_{a}\right),
\end{gathered}
$$

и

$$
\begin{gathered}
\mathcal{F}_{12}^{\hbar, a}(u)=e^{2 \pi i N \hbar \partial_{\tau} \Omega_{a}} F_{12}^{\hbar}\left(u+N \Omega_{a}\right), \\
\mathcal{F}_{21}^{\hbar, a}(-u)=e^{-2 \pi i N \hbar \partial_{\tau} \Omega_{a}} F_{21}^{\hbar}\left(-u-N \Omega_{a}\right) ;
\end{gathered}
$$

здесь

$$
F_{a b}^{\hbar}(u)=\partial_{u} R_{a b}^{\hbar}(u) .
$$

Матрицы $L(\hbar), M(\hbar) \in \operatorname{Mat}(2, \mathbb{C}) \otimes \operatorname{Mat}(N, \mathbb{C})^{\otimes 2}$. Их размер равен $2 N^{2} \times 2 N^{2}$.

Уравнение Пенлеве VI (1.60) эквивалентно уравнению, сохраняющему монодромию:

$$
\frac{d}{d \tau} L(\hbar)-\frac{1}{2 \pi i} \frac{d}{d \hbar} M(\hbar)=[L(\hbar), M(\hbar)],
$$

где постоянная Планка $\hbar$ играет роль спектрального параметра (см. работу [1]).

Для $N=1$ ответ (1.61), (1.62) воспроизводит эллиптическую $(2 \times 2)$-пару Лакса, предложенную в работе [14]. Пара Лакса (1.61), (1.62) работает и для четных $N$. Но в этом случае уравнение Пенлеве содержит только одну константу:

$$
\frac{d^{2} u}{d \tau^{2}}=-\nu^{2} \wp^{\prime}(u), \quad \nu^{2}=\sum_{a=0}^{3} \nu_{a}^{2} .
$$

\footnotetext{
4) Коэффициент $1 / \sqrt{-2}$ дает нормировку константы связи, как в (1.60).
} 


\section{2. ДВОЙНОЙ РЯД КРОНЕКЕРА \\ И R-МАТРИЦА БАКСТЕРА-БЕЛАВИНА}

Следуя идее работы [2], получим $R$-матрицу Бакстера-Белавина в виде обобщения ряда Кронекера.

(. $\$ \mathrm{R} \$$ матрица в переменных Якоби) $R$-матрица в переменных Якоби Представим эллипти $\Sigma_{\tau}(1.4)$ в форме Якоби:

$$
C_{q}=\mathbb{C} / q^{\mathbb{Z}}, \quad q=\mathbf{e}(\tau)=e^{2 \pi i \tau} .
$$

Рассмотрим произведение $C_{q} \times C_{q}$ с координатами $s=\mathbf{e}(u), t=\mathbf{e}(z)$. Вместо функции Кронекера $\phi(z, u)$ будем рассматривать распределение $g(s, t)$ на пространстве полиномов Лорана $\mathbb{C}\left[\left[s^{-1}, t^{-1}, s, t\right]\right]$. Для $|q|<|t|<1$ оно представляется в виде ряда

$$
g(s, t \mid q)=\sum_{n \in \mathbb{Z}} \frac{t^{n}}{q^{n} s-1} .
$$

Если при этом также $|q|<|s|<1$, то

$$
\begin{gathered}
g(s, t \mid q)=-g^{+}(s, t \mid q)+g^{-}(s, t \mid q), \\
g^{+}(s, t \mid q)=\sum_{i, n \geqslant 0} s^{i} q^{i n} t^{n}, \quad g^{-}(s, t \mid q)=\sum_{i, n<0} s^{i} q^{i n} t^{n}
\end{gathered}
$$

или, эквиваленто,

$$
g(s, t \mid q)=1-\frac{1}{1-t}-\frac{1}{1-s}+g^{-}(s, t)-\sum_{i, n>0} s^{i} q^{i n} t^{n} .
$$

В области $|q|<|t|<1$ и $|q|<|s|<1$ имеем

$$
\left.g(s, t \mid q)\right|_{s=\frac{\ln u}{2 \pi i}, t=\frac{\ln z}{2 \pi i}}=\phi(z, u) .
$$

Распределение $g(s, t \mid q)$ обладает свойствами, аналогичными (1.6)-(1.9). В частности,

$$
g(s, t \mid q)=g(t, s \mid q)
$$

Из (2.2) следует, что

$$
g\left(s^{-1}, t^{-1} \mid q\right)=-g(s, t \mid q)+\delta(t)+\delta(s)-2,
$$

где $\delta(s)$ - распределение на пространстве $\mathbb{C}\left[t, t^{-1}\right]=\left\{\psi(t)=\sum_{l} c_{l} t^{l}\right\}$, заданное функционалом $\langle\delta, \psi\rangle=\operatorname{Res}_{t=0} \psi(t)$ и представимое в виде формального ряда

$$
\delta(t)=\sum_{n \in \mathbb{Z}} t^{n}
$$

Аналог квазипериодических свойств (1.11) появляется следующим образом. Распределение $g(s, t)$ есть решение следующего разностного уравнения по $t$ (функция Грина):

$$
s g(s, t q \mid q)-g(s, t \mid q)=\delta(t)-1 .
$$


Оно задает продолжение распределения $g(s, t \mid q)$ из области $|q|<|t|<1$ на $\mathbb{C}^{*}$. В силу (2.5) аналогичное уравнение имеет место и по переменной $s$.

Пусть $\eta=\mathbf{e}(\hbar)$. $R$-матрица (1.24) в переменных $(s, t, \eta)$ принимает следующий вид:

$$
\begin{aligned}
R_{12}^{\hbar}(s) & =\sum_{\alpha \in \mathbb{Z}_{N} \times \mathbb{Z}_{N}} s^{\alpha_{2} / N} g\left(s, \omega_{\alpha}+\hbar\right) T_{\alpha} \otimes T_{-\alpha}= \\
& =\sum_{\alpha \in \mathbb{Z}_{N} \times \mathbb{Z}_{N}} s^{\alpha_{2} / N}\left(\sum_{m, n} \mathbf{e}\left(\frac{n \alpha_{1}}{N}\right) q^{n\left(m+\alpha_{2} / N\right)} \eta^{n} s^{m}\right) T_{\alpha} \otimes T_{-\alpha} .
\end{aligned}
$$

Она играет роль функции Грина разностного оператора:

$$
\eta \Lambda \otimes 1 R_{12}^{\hbar}(s q) \Lambda^{-1} \otimes 1-R_{12}^{\hbar}(s)=(\delta(s)-1) P_{12} .
$$

Двойной ряд Кронекера [8]. Распределение $g(s, t \mid q)($ и $\phi(z, u))$ представляется в виде двойного ряда Кронекера. Рассмотрим решетку на комплексной плоскости $\mathbb{C}$ :

$$
W=\{\gamma=m+n \tau, m, n \in \mathbb{Z}\}
$$

Запишем аргумент $u$ функции $\phi(z, u)$ в виде $u=u_{1}+u_{2} \tau$ (здесь $u_{1}, u_{2}$ вещественные), и пусть $\chi_{u}(\gamma)=\mathbf{e}\left(-m u_{2}+n u_{1}\right)$ есть характер решетки $W\left(\chi_{u}(\gamma): W \rightarrow S^{1}\right)$, зависящий от $u \in \Sigma_{\tau}$. Двойной ряд Кронекера задается как

$$
S(z, u \mid \tau)=\sum_{\gamma \in W} \frac{\chi_{u}(\gamma)}{z+\gamma}
$$

Из определения следует, что

$$
\begin{aligned}
& S(z+1, u \mid \tau)=\mathbf{e}\left(u_{2}\right) S(z, u \mid \tau), \\
& S(z+\tau, u \mid \tau)=\mathbf{e}\left(-u_{1}\right) S(z, u \mid \tau) .
\end{aligned}
$$

В монографии [8] доказано, что $S(z, u \mid \tau)$ выражается через функцию Кронекера следующим образом:

$$
S(z, u \mid \tau)=\mathbf{e}\left(u_{2} z\right) \phi(z, u)
$$

или, в координатах Якоби,

$$
S(t, s \mid q)=t^{u_{2}} g(s, t \mid q)
$$

Перейдем теперь к $R$-матрице и опишем ее тоже в виде двойного ряда Кронекера $S(z, u \mid \tau)(2.11)$. Зададим решетку $W$ двумя генераторами $\left(\alpha_{1} / N+\hbar_{1},\left(\alpha_{2} / N+\hbar_{2}\right) \tau\right)$, где $\hbar=\hbar_{1}+\hbar_{2} \tau, \hbar_{1,2} \in \mathbb{R}$. Соответствующий характер решетки $W$ имеет вид

$$
\chi_{(m, n)}(\alpha, \hbar)=\mathbf{e}\left(-m\left(\frac{\alpha_{2}}{N}+\hbar_{2}\right)+n\left(\frac{\alpha_{1}}{N}+\hbar_{1}\right)\right) .
$$

Тогда $R$-матрица (1.24) записывается через (2.11):

$$
R_{12}^{\hbar}(z)=\mathbf{e}\left(-\hbar_{2} z\right) \sum_{(m, n) \in \mathbb{Z} \oplus \mathbb{Z}} \frac{\sum_{\alpha \in \mathbb{Z}_{N} \times \mathbb{Z}_{N}} \chi_{(m, n)}(\alpha, \hbar) T_{\alpha} \otimes T_{-\alpha}}{z+m+n \tau} .
$$


Квазипериодические свойства (1.40), (1.41) становятся очевидными. Из формулы (2.13) следует, что особенность при $z, \hbar \rightarrow 0$ удовлетворяет равенствам (1.36).

Перейдем от $R_{12}^{\hbar}(z)$ к

$$
\widetilde{R}_{12}^{\hbar}(z)=\mathbf{e}\left(\hbar_{2} z\right) R_{12}^{\hbar}(z) .
$$

Это выражение тоже удовлетворяет уравнению Янга-Бакстера, а квазипериодические свойства приобретают вид (ср. с (1.40))

$$
\begin{aligned}
& \widetilde{R}_{12}^{\hbar}(z+1)=\mathbf{e}\left(\hbar_{2}\right) Q^{-1} \otimes 1 \widetilde{R}_{12}^{\hbar}(z) Q \otimes 1, \\
& \widetilde{R}_{12}^{\hbar}(z+\tau)=\mathbf{e}\left(\hbar_{1}\right) \Lambda^{-1} \otimes 1 \widetilde{R}_{12}^{\hbar}(z) \Lambda \otimes 1 .
\end{aligned}
$$

В отличие от свойств (1.41), матрица $\widetilde{R}$ не является голоморфной по $\hbar$, но является двоякопериодической.

ЗАмечАниЕ 1. Представление (2.16) означает, что эллиптическая $\widetilde{R}$-матрица представляется в виде усреднения матрицы Янга $z^{-1} P_{12}$ по решетке $W$, подкрученной на характер (2.15).

Из (1.30) следует представление и для классической $r$-матрицы:

$$
\begin{aligned}
r_{12}(z)= & E_{1}(z) 1 \otimes 1+\sum_{(m, n) \in(\mathbb{Z} \oplus \mathbb{Z}) \backslash(0,0)} \frac{\sum_{\alpha \in \mathbb{Z}_{N} \times \mathbb{Z}_{N}} \chi_{(m, n)}(\alpha, 0) T_{\alpha} \otimes T_{-\alpha}}{z+m+n \tau}, \\
m_{12}(z)= & \frac{E_{1}^{2}(z)-\wp(z)}{2} 1 \otimes 1+ \\
& +\sum_{(m, n) \in(\mathbb{Z} \oplus \mathbb{Z}) \backslash(0,0)} \frac{\sum_{\alpha \in \mathbb{Z}_{N} \times \mathbb{Z}_{N}}(z+m+n \bar{\tau}) \chi_{(m, n)}(\alpha, 0) T_{\alpha} \otimes T_{-\alpha}}{(z+m+n \tau)(\bar{\tau}-\tau)} .
\end{aligned}
$$

\section{3. ВЫВОД ТОЖДЕСТВ}

Имеют место следующие утверждения.

ПрЕДЛОЖЕНИЕ 3.1. R-матрица (1.24) удовлетворяет свойству симметрии аргументов (1.29).

ДокАЗАТЕЛьСтво. Используя определения (1.28) и (1.23), имеем

$$
\begin{aligned}
R_{12}^{z / N}(N \hbar) P_{12} & =\frac{1}{N} \sum_{\alpha, \beta} T_{\alpha} T_{\beta} \otimes T_{-\alpha} T_{-\beta} \varphi_{\alpha}\left(N \hbar, \omega_{\alpha}+\frac{z}{N}\right)= \\
& =\frac{1}{N} \sum_{\alpha, \beta} \kappa_{\alpha, \beta}^{2} T_{\alpha+\beta} \otimes T_{-\alpha-\beta} \varphi_{\alpha}\left(N \hbar, \omega_{\alpha}+\frac{z}{N}\right) .
\end{aligned}
$$

Так как $\kappa_{\alpha, \beta}=\kappa_{\alpha, \alpha+\beta}$, свойство (1.29) эквивалентно набору $N^{2}$ скалярных тождеств

$$
\frac{1}{N} \sum_{\alpha} \kappa_{\alpha, \gamma}^{2} \varphi_{\alpha}\left(N \hbar, \omega_{\alpha}+\frac{z}{N}\right)=\varphi_{\gamma}\left(z, \omega_{\gamma}+\hbar\right), \quad \gamma \in \mathbb{Z} \times \mathbb{Z},
$$

или

$$
\frac{1}{N} \sum_{\alpha} \kappa_{\alpha, \gamma}^{2} \varphi_{\alpha}\left(z, \omega_{\alpha}+\hbar\right)=\varphi_{\gamma}\left(N \hbar, \omega_{\gamma}+\frac{z}{N}\right), \quad \gamma \in \mathbb{Z} \times \mathbb{Z}
$$


Проверка этих тождеств производится путем сравнения полюсов и вычетов. Нам также потребуется выражение для суммы корней $N$-й степени из единицы (оно следует в том числе из $\left.P_{12}^{2}=1\right)$ :

$$
\sum_{\alpha} \kappa_{\alpha, \gamma}^{2}=N^{2} \delta_{\gamma, 0}
$$

Вычислим вычет обеих частей равенства (3.2) в точке $\hbar=-\omega_{\mu}$. В правой части получим $\delta_{\mu, \gamma} e^{2 \pi i \partial_{\tau} \omega_{\gamma} z}$ в силу (1.8). В левой части имеем

$$
\begin{gathered}
\operatorname{Res}_{\hbar=-\omega_{\mu}} \frac{1}{N} \sum_{\alpha} \kappa_{\alpha, \gamma}^{2} \varphi_{\alpha}\left(N \hbar, \omega_{\alpha}+\frac{z}{N}\right)= \\
=\operatorname{Res}_{\hbar=0} \frac{1}{N} \sum_{\alpha} \kappa_{\alpha, \gamma}^{2} \varphi_{\alpha}\left(N \hbar-N \omega_{\mu}, \omega_{\alpha}+\frac{z}{N}\right) \stackrel{(1.10),(1.11)}{=} \\
\stackrel{(1.10),(1.11)}{=} \operatorname{Res}_{\hbar=0} \frac{1}{N} \sum_{\alpha} \kappa_{\alpha, \gamma}^{2} \kappa_{\alpha,-\mu}^{2} e^{2 \pi i \partial_{\tau} \omega_{\mu} z} \varphi_{\alpha}\left(N \hbar, \omega_{\alpha}+\frac{z}{N}\right) \stackrel{(1.8)}{=} \\
\stackrel{(1.8)}{=} \frac{1}{N} \sum_{\alpha} \kappa_{\alpha, \gamma-\mu}^{2} e^{2 \pi i \partial_{\tau} \omega_{\mu} z} \frac{1}{N} \stackrel{(3.4)}{=} \delta_{\mu, \gamma} e^{2 \pi i \partial_{\tau} \omega_{\mu} z}
\end{gathered}
$$

ПрЕДЛОЖЕНИЕ 3.2. R-матрица (1.24) удовлетворяет (1.52)- тождеству Фэя в пространстве $\operatorname{Mat}(N, \mathbb{C})^{\otimes 2}$.

ДоказАтЕЛьство. Рассмотрим выражение

$$
R_{12}^{\hbar}(z) R_{21}^{\hbar^{\prime}}(-w)=-\sum_{\alpha, \beta} \kappa_{\alpha, \beta}^{2} T_{\alpha+\beta} \otimes T_{-\alpha-\beta} \varphi_{\alpha}\left(z, \omega_{\alpha}+\hbar\right) \varphi_{\beta}\left(w, \omega_{\beta}-\hbar^{\prime}\right) .
$$

Здесь уже использовано свойство $R_{21}^{\hbar^{\prime}}(-w)=-R_{12}^{-\hbar^{\prime}}(w)$. Применим поочередно тождество Фэя (1.15), затем (3.3), а затем снова (1.15):

$$
\begin{aligned}
& R_{12}^{\hbar}(z) R_{21}^{\hbar^{\prime}}(-w)= \\
&=-\sum_{\alpha, \beta} \kappa_{\alpha, \beta}^{2} T_{\alpha+\beta} \otimes T_{-\alpha-\beta} \varphi_{\alpha}\left(z-w, \omega_{\alpha}+\hbar\right) \varphi_{\alpha+\beta}\left(w, \omega_{\alpha+\beta}+\hbar-\hbar^{\prime}\right)- \\
&-\sum_{\alpha, \beta} \kappa_{\alpha, \beta}^{2} T_{\alpha+\beta} \otimes T_{-\alpha-\beta} \varphi_{\beta}\left(w-z, \omega_{\beta}-\hbar^{\prime}\right) \varphi_{\alpha+\beta}\left(z, \omega_{\alpha+\beta}+\hbar-\hbar^{\prime}\right)= \\
&=-N \sum_{\gamma} T_{\gamma} \otimes T_{-\gamma} \varphi_{\gamma}\left(N \hbar, \omega_{\gamma}+\frac{z-w}{N}\right) \varphi_{\gamma}\left(w, \omega_{\gamma}+\hbar-\hbar^{\prime}\right)+ \\
&+N \sum_{\gamma} T_{\gamma} \otimes T_{-\gamma} \varphi_{\gamma}\left(N \hbar^{\prime}, \omega_{\gamma}+\frac{z-w}{N}\right) \varphi_{\gamma}\left(z, \omega_{\gamma}+\hbar-\hbar^{\prime}\right)= \\
&= N \sum_{\gamma} T_{\gamma} \otimes T_{-\gamma}\left(-\phi\left(N \hbar, \frac{z-w}{N}+\hbar^{\prime}-\hbar\right) \varphi_{\gamma}\left(w+N \hbar, \omega_{\gamma}+\hbar-\hbar^{\prime}\right)-\right. \\
&-\phi\left(w, \hbar-\hbar^{\prime}-\frac{z-w}{N}\right) \varphi_{\gamma}\left(w+N \hbar, \omega_{\gamma}+\frac{z-w}{N}\right)+ \\
&+\phi\left(N \hbar^{\prime}, \frac{z-w}{N}+\hbar^{\prime}-\hbar\right) \varphi_{\gamma}\left(z+N \hbar^{\prime}, \omega_{\gamma}+\hbar-\hbar^{\prime}\right)+ \\
&\left.+\phi\left(z, \hbar-\hbar^{\prime}-\frac{z-w}{N}\right) \varphi_{\gamma}\left(z+N \hbar^{\prime}, \omega_{\gamma}+\frac{z-w}{N}\right)\right) .
\end{aligned}
$$


ПРЕДЛОЖЕНИЕ 3.3. R-матрицы (1.24) и (1.31) удовлетворяют вырожденным тождествам Фэя $(1.55),(1.56)$ в пространстве $\operatorname{Mat}(N, \mathbb{C})^{\otimes 2}$.

ДокаЗАтельство. Начнем с тождества (1.55). Рассмотрим выражение

$$
R_{12}^{\hbar}(z) R_{21}^{\hbar}(-w)=-\sum_{\alpha, \beta} \kappa_{\alpha, \beta}^{2} T_{\alpha+\beta} \otimes T_{-\alpha-\beta} \varphi_{\alpha}\left(z, \omega_{\alpha}+\hbar\right) \varphi_{\beta}\left(w, \omega_{\beta}-\hbar\right)
$$

Разобьем его на две части в соответствии с $\sum_{\alpha, \beta}=\sum_{\alpha \neq-\beta}+\sum_{\alpha=-\beta}$. Первую часть преобразуем, как в предложении 3.2 (с использованием (1.15), затем (3.3) и снова (1.15)):

$$
\begin{aligned}
&-\sum_{\alpha \neq-\beta} \kappa_{\alpha, \beta}^{2} T_{\alpha+\beta} \otimes T_{-\alpha-\beta} \varphi_{\alpha}\left(z, \omega_{\alpha}+\hbar\right) \varphi_{\beta}\left(w, \omega_{\beta}-\hbar\right)= \\
&=-\sum_{\alpha \neq-\beta} \kappa_{\alpha, \beta}^{2} T_{\alpha+\beta} \otimes T_{-\alpha-\beta} \varphi_{\alpha}\left(z-w, \omega_{\alpha}+\hbar\right) \varphi_{\alpha+\beta}\left(w, \omega_{\alpha+\beta}\right)- \\
&-\sum_{\alpha \neq-\beta} \kappa_{\alpha, \beta}^{2} T_{\alpha+\beta} \otimes T_{-\alpha-\beta} \varphi_{\beta}\left(w-z, \omega_{\beta}-\hbar\right) \varphi_{\alpha+\beta}\left(z, \omega_{\alpha+\beta}\right)= \\
&= \cdots=-N \phi\left(\frac{z-w}{N}, N \hbar\right) \sum_{\gamma \neq 0} T_{\gamma} \otimes T_{-\gamma} \varphi_{\gamma}\left(w+N \hbar, \omega_{\gamma}\right)- \\
&-N \phi\left(\frac{w-z}{N}, w\right) \sum_{\gamma \neq 0} T_{\gamma} \otimes T_{-\gamma} \varphi_{\gamma}\left(w+N \hbar, \omega_{\gamma}+\frac{z-w}{N}\right)+ \\
&+N \phi\left(\frac{z-w}{N}, N \hbar\right) \sum_{\gamma \neq 0} T_{\gamma} \otimes T_{-\gamma} \varphi_{\gamma}\left(z+N \hbar, \omega_{\gamma}\right)+ \\
&+N \phi\left(\frac{w-z}{N}, z\right) \sum_{\gamma \neq 0} T_{\gamma} \otimes T_{-\gamma} \varphi_{\gamma}\left(z+N \hbar, \omega_{\gamma}+\frac{z-w}{N}\right) .
\end{aligned}
$$

Добавляя (и вычитая) скалярные члены $1 \otimes 1$ в каждой строке, получим первую и вторую строки тождества (1.55). K скалярным частям добавляется также выражение

$$
\begin{aligned}
& -\sum_{\alpha=-\beta} \kappa_{\alpha, \beta}^{2} T_{\alpha+\beta} \otimes T_{-\alpha-\beta} \varphi_{\alpha}\left(z, \omega_{\alpha}+\hbar\right) \varphi_{\beta}\left(w, \omega_{\beta}-\hbar\right)= \\
& =1 \otimes 1 \sum_{\alpha} \varphi_{\alpha}\left(z, \omega_{\alpha}+\hbar\right) \varphi_{\alpha}\left(-w, \omega_{\alpha}+\hbar\right) \stackrel{(1.17)}{=} \\
& \stackrel{(1.17)}{=} 1 \otimes 1 \sum_{\alpha} \varphi_{\alpha}\left(z-w, \omega_{\alpha}+\hbar\right)\left(E_{1}(z)-E_{1}(w)+E_{1}\left(\hbar+\omega_{\alpha}\right)-E_{1}\left(z-w+\hbar+\omega_{\alpha}\right)\right) .
\end{aligned}
$$

Оно преобразуется с помощью равенства (3.3) для $\gamma=0$,

$$
\sum_{\alpha} \varphi_{\alpha}\left(z-w, \omega_{\alpha}+\hbar\right)=N \phi\left(N \hbar, \frac{z-w}{N}\right)
$$


и производной $(1.13),(1.14)$ от него же по $\hbar$ :

$$
\begin{gathered}
\sum_{\alpha} \varphi_{\alpha}\left(z-w, \omega_{\alpha}+\hbar\right)\left(E_{1}\left(z-w+\hbar+\omega_{\alpha}\right)-E_{1}\left(\hbar+\omega_{\alpha}\right)\right)= \\
=N^{2} \phi\left(N \hbar, \frac{z-w}{N}\right)\left(E_{1}\left(N \hbar+\frac{z-w}{N}\right)-E_{1}(N \hbar)\right) .
\end{gathered}
$$

Таким образом завершается доказательство тождества (1.55). Тождество (1.56) доказывается аналогично. Кроме того, (1.56) выводится из (1.55) с использованием (1.29) и (1.37).

\section{4. МНОГОМЕРНЫЕ ЭЛЛИПТИЧЕСКИЕ ПАРЫ ЛАКСА ДЛЯ УРАВНЕНИЯ ПЕНЛЕВЕ VI}

Для уравнений Пенлеве известны матричные пары Лакса разного типа и размера (см., например, работы [15]-[17]). В этом разделе мы построим $R$-матричнозначное обобщение эллиптической $(2 \times 2)$-пары Лакса, предложенной в статье [14].

ПреДлОЖенИЕ 4.1. Уравнение Пенлеве VI в эллиптической форме (1.60) эквивалентно изомонодромным уравнениям (1.66) с парой Лакса (1.61)-(1.65) и эллиптической $R$-матричей (1.24) для нечетных $N$.

ДокАзАТЕЛЬСтво аналогично предложенному в [14] для скалярного $(N=1)$ случая.

Во-первых, заметим, что

$$
\frac{d}{d \tau} L(\hbar)=\frac{d u}{d \tau} \partial_{u} L(\hbar)+\partial_{\tau} L(\hbar)
$$

где последнее слагаемое есть производная по явной зависимости от $\tau$. Это слагаемое сокращается с величиной $\frac{1}{2 \pi i} \frac{d}{d \hbar} M(\hbar)$ в силу уравнения теплопроводности (1.48) $2 \pi i \partial_{\tau} \mathcal{R}_{b c}^{\hbar, a}(u)=\partial_{\hbar} \mathcal{F}_{b c}^{\hbar, a}(u)$.

Положим

$$
L^{a}=\left(\begin{array}{cc}
0 & \mathcal{R}_{12}^{\hbar, a}(u) \\
\mathcal{R}_{21}^{\hbar, a}(-u) & 0
\end{array}\right), \quad M^{a}=\left(\begin{array}{cc}
0 & \mathcal{F}_{12}^{\hbar, a}(u) \\
\mathcal{F}_{21}^{\hbar, a}(-u) & 0
\end{array}\right)
$$

Основное утверждение, необходимое нам для проверки, состоит в том, что для $a \neq b$

$$
\left[L^{a}, M^{b}\right]+\left[L^{b}, M^{a}\right]=0
$$

т. е. вклад в $[L(\hbar), M(\hbar)]$ дают только члены вида $\left[L^{a}, M^{a}\right]$. Действительно, из унитарности (1.27) следует, что

$$
\mathcal{R}_{12}^{\hbar, a}(u) \mathcal{R}_{21}^{\hbar, a}(-u)=R_{12}^{\hbar}\left(u+N \Omega_{a}\right) R_{21}^{\hbar}\left(-u-N \Omega_{a}\right)=N^{2}\left(\wp(N \hbar)-\wp\left(u+N \Omega_{a}\right)\right) .
$$

Беря производную (4.3) по $u$, получим

$$
\mathcal{F}_{12}^{\hbar, a}(u) \mathcal{R}_{21}^{\hbar, a}(-u)-\mathcal{R}_{12}^{\hbar, a}(u) \mathcal{F}_{21}^{\hbar, a}(-u)=-N^{2} \wp^{\prime}\left(u+N \Omega_{a}\right)
$$

Это тождество дает уравнение движения. Заметим, что для наличия четырех произвольных констант число $N$ должно быть нечетным, так как в этом случае мы 
имеем $\wp^{\prime}\left(u+N \Omega_{a}\right)=\wp^{\prime}\left(u+\Omega_{a}\right)$. Если же $N$ - четное число, то $\wp^{\prime}\left(u+N \Omega_{a}\right)=\wp^{\prime}(u)$, и остается только одна константа, как в (1.67).

Для доказательства равенства (4.2) напомним, что в скалярном случае оно следует из соотношения

$$
\begin{aligned}
\varphi_{a}(\hbar, u & \left.+\Omega_{a}\right) f_{b}\left(\hbar,-u-\Omega_{b}\right)-f_{b}\left(\hbar, u+\Omega_{b}\right) \varphi_{a}\left(\hbar,-u-\Omega_{a}\right)+ \\
& +\varphi_{b}\left(\hbar, u+\Omega_{b}\right) f_{a}\left(\hbar,-u-\Omega_{a}\right)-f_{a}\left(\hbar, u+\Omega_{a}\right) \varphi_{b}\left(\hbar,-u-\Omega_{b}\right)=0,
\end{aligned}
$$

где функция

$$
f_{a}\left(z, u+\Omega_{a}\right)=\left.e^{2 \pi i \partial_{\tau} \Omega_{a} \hbar} \partial_{w} \phi(\hbar, w)\right|_{w=u+\Omega_{a}}
$$

есть скалярный аналог $\mathcal{F}_{12}^{\hbar, a}(u)$. Тождество $(4.5)$ выводится из $(1.16)$ и $(1.10),(1.11)$ следующим образом:

$$
\begin{aligned}
& \varphi_{a}\left(\hbar, u+\Omega_{a}\right) \varphi_{b}\left(\hbar,-u-\Omega_{b}\right)+\varphi_{b}\left(\hbar, u+\Omega_{b}\right) \varphi_{a}\left(\hbar,-u-\Omega_{a}\right)= \\
& \quad=\varphi_{a+b}\left(\hbar, \Omega_{a}+\Omega_{b}\right)\left(2 E_{1}(\hbar)-E_{1}\left(\hbar+\Omega_{a}-\Omega_{b}\right)-E_{1}\left(\hbar+\Omega_{b}-\Omega_{a}\right)\right) .
\end{aligned}
$$

Правая часть этого равенства не зависит от $u$. Производная соотношения (4.6) по $u$ дает (4.5).

Аналогично (4.6) из вырожденного тождества Фэя (1.55) следует равенство

$$
\begin{aligned}
& \mathcal{R}_{12}^{\hbar, a}(u) \mathcal{R}_{21}^{\hbar, b}(-u)+\mathcal{R}_{12}^{\hbar, b}(u) \mathcal{R}_{21}^{\hbar, a}(-u)= \\
& =N^{2} 1 \otimes 1 \varphi_{a+b}\left(N \hbar, \Omega_{a}+\Omega_{b}\right)\left(2 E_{1}(N \hbar)-E_{1}\left(N \hbar+\Omega_{a}-\Omega_{b}\right)-E_{1}\left(N \hbar+\Omega_{b}-\Omega_{a}\right)\right) .
\end{aligned}
$$

Его можно непосредственно проверить, используя соотношения (1.10), (1.11), которые переписываются в виде

$$
\phi\left(z, w+\Omega_{a}\right)=e^{-2 \pi i z \partial_{\tau} \Omega_{a}} \phi\left(z, w-\Omega_{a}\right) .
$$

Правая часть равенства (4.7) является скалярной величиной и не зависит от $u$. Производная от (4.7) по $u$ дает

$$
\mathcal{F}_{12}^{\hbar, a}(u) \mathcal{R}_{21}^{\hbar, b}(-u)-\mathcal{R}_{12}^{\hbar, a}(u) \mathcal{F}_{21}^{\hbar, b}(-u)+\mathcal{F}_{12}^{\hbar, b}(u) \mathcal{R}_{21}^{\hbar, a}(-u)-\mathcal{R}_{12}^{\hbar, b}(u) \mathcal{F}_{21}^{\hbar, a}(-u)=0 .
$$

Это тождество лежит в основе (4.2).

Благодарности. Мы выражаем благодарность Н. Славнову и А. Забродину за ценные замечания. Исследование А.В. Зотова (результаты разделов 3,4 ) выполнено за счет гранта Российского научного фонда (проект 14-50-00005) в Математическом институте им. В. А. Стеклова РАН.

\section{Список литературы}

[1] A. Levin, M. Olshanetsky, A. Zotov, JHEP, 10 (2014), 109, 29 pp., arXiv: 1408.6246.

[2] A. Polischuk, Adv. Math., 168:1 (2002), 56-95.

[3] R. J. Baxter, Ann. Phys., 70 (1972), 193-228.

[4] A. A. Belavin, Nucl. Phys. B, 180:2 (1981), 189-200; А. А. Белавин, В. Г. Дринфельд, Функи. анализ и его прил., 16:3 (1982), 1-29. 
[5] M. P. Richey, C. A. Tracy, J. Statist. Phys., 42:3-4 (1986), 311-348; K. Hasegawa, J. Math. Phys., 35:11 (1994), 6158-6171.

[6] V. V. Bazhanov, Y. G. Stroganov, "On connection between the solutions of the quantum and classical triangle equations", Проблемы физики высоких энергий и квантовой теории поля, 1, Ин-т физики высоких энергий, Протвино, 1983, 52-54.

[7] Л. А. Тахтаджян, Зап. научн. сем. ЛОМИ, 133 (1984), 258-276.

[8] A. Weil, Elliptic Functions According to Eisenstein and Kronecker, Ergebnisse der Mathematik und ihrer Grenzgebiete, 88, Springer, Berlin, New York, 1976.

[9] Д. Мамфорд, Лекиии о тэта-функииях, Мир, М., 1988.

[10] J. D. Fay, Theta Functions on Riemann Surfaces, Lecture Notes in Mathematics, 352, Springer, Berlin, 1973.

[11] A. Levin, M. Olshanetsky, A. Zotov, JHEP, 07 (2014), 012, 38 pp., arXiv: 1405.7523; Nucl. Phys. B, 887 (2014), 400-422, arXiv: 1406.2995.

[12] M. Aguiar, "Infinitesimal Hopf algebras", New Trends in Hopf Algebra Theory (La Falda, Sierras de Córdoba, Argentina, August 9-13, 1999), Contemporary Mathematics, 267, eds. N. Andruskiewitsch, W. R. Ferrer Santos, H.-J. Schneider, AMS, Providence, RI, 2000, $1-29$.

[13] P. Painlevé, C. R. Acad. Sci. Paris, 143 (1906), 1111-1117; Yu. Manin, "Sixth Painlevé equation, universal elliptic curve, and mirror of $\mathbb{P}^{2} "$, Geometry of Differential Equations, Translations American Mathematical Society. Ser. 2, 186, Advances in the Mathematical Sciences, 39, eds. A. Khovanskij, A. Varchenko, V. Vassiliev, AMS, Providence, RI, 1998, $131-151$

[14] A. Zotov, Lett. Math. Phys., 67:2 (2004), 153-165, arXiv: hep-th/0310260.

[15] M. Jimbo, T. Miwa, Phys. D, 2:3 (1981), 407-448; N. Joshi, A. Kitaev, P. Treharne, J. Math. Phys., 48:10 (2007), 103512, 42 pp., arXiv:0706.1750; M. Noumi, Y. Yamada, "A new Lax pair for the sixth Painlevé equation associated with $\widehat{s o}(8)$ ", Microlocal Analysis and Complex Fourier Analysis, eds. T. Kawai, K. Fujita, World Sci., Singapore, 2002, 238-252, arXiv: math-ph/0203029; G. Aminov, S. Arthamonov, New $2 \times 2$-matrix linear problems for Painlevé equations, arXiv: 1112.4688.

[16] B. Dubrovin, M. Mazzocco, Invent. Math., 141:1 (2000), 55-147, arXiv: math/9806056; P. Boalch, Proc. London Math. Soc. (3), 90:1 (2005), 167-208.

[17] A. Levin, M. Olshanetsky, "Painlevé-Calogero correspondence", Calogero-Moser-Sutherland Models (Montréal, QC, 1997), CRM Series in Mathematical Physics, eds. J.F. van Diejen, L. Vinet, Springer, New York, 2000, 313-332, arXiv: alg-geom/9706010; A. Levin, M. Olshanetsky, A. Zotov, Commun. Math. Phys., 268:1 (2006), 67-103, arXiv: math/0508058; A. Levin, A. Zotov, "On rational and elliptic forms of Painlevé VI equation", Moscow Seminar on Mathematical Physics. II, Translations American Mathematical Society. Ser. 2, 221, Advances in the Mathematical Sciences, 60, eds. Yu. Neretin, M. A. Olshanetsky, A. Rosly, AMS, Providence, RI, 2007, 173-184; A. Zabrodin, A. Zotov, J. Math. Phys., 53:7 (2012), 073507, 19 pp., arXiv: 1107.5672; 073508, 19 pp.; Construct. Approx., 41:3 (2015), 325-423, arXiv: 1212.5813; А. М. Левин, М. А. Ольшанецкий, А. В. Зотов, УМH, 69:1(415) (2014), 39-124, arXiv: 1311.4498. 